\title{
Posterpreise 2012
}

1. Preis V. Venkataramani', K. Thiele², C.-L. Behnes², G. G. Wulf', P. Thelen ${ }^{3}$, L. Opitz ${ }^{4}$,

G. Salinas-Riester ${ }^{4}$, O. Wirths ${ }^{5}$, T. A. Bayer ${ }^{5}$, H.-J. Radzun², S. Schweyer ${ }^{2}$

${ }^{\top}$ Abteilung Hämatologie und Onkologie, Universitätsmedizin Göttingen

${ }^{2}$ Abteilung Pathologie, Universitätsmedizin Göttingen

${ }^{3}$ Abteilung Urologie, Universitätsmedizin Göttingen

${ }^{4}$ DNA Microarray Facility, Universitätsmedizin Göttingen

${ }^{5}$ Division Molekulare Psychiatrie, Universitätsmedizin Göttingen

Das Amyloid-Vorläufer-Protein (APP) ist ein neuer Biomarker für transformierte humane pluripotente Stammzellen

2. Preis H. Reis ${ }^{1,2}$, F. vom Dorp ${ }^{3}$, C. Niedworok ${ }^{3}$, A. Melchior-Becker ${ }^{4}$,

J. W. Fischer ${ }^{4}$, D. Gödde', B. B. Singer ${ }^{5}$, A. Bankfalvi' ${ }^{2}$, I. Romics ${ }^{6}$, K. W. Schmid ${ }^{2}$,

S. Störkel' ${ }^{1}$, S. Ergün ${ }^{5}$, H. Rübben 3 , T. Szarvas ${ }^{3,7}$

${ }^{1}$ Institut für Pathologie, Universität Witten/Herdecke, Wuppertal

${ }^{2}$ Institut für Pathologie und Neuropathologie, Universität Duisburg-Essen, Essen

${ }^{3}$ Klinik für Urologie, Universität Duisburg-Essen, Essen

${ }_{4}^{4}$ Institut für Pharmakologie und klinische Pharmakologie, Universität Duisburg-Essen, Essen

IInstitut für Anatomie, Universität Duisburg-Essen, Essen

${ }^{6} \mathrm{Klinik}$ für Urologie, Semmelweis Universität, Budapest, Ungarn

${ }^{7}$ Klinik für Urologie, Medizinische Universität Wien, Österreich

Eine hohe IMP3-Proteinexpression ist ein negativer prognostischer Faktor bei

fortgeschrittenem Urothelkarzinom der Harnblase

3. Preis T. T. Rau' ${ }^{1}$, A. Agaimy ${ }^{2}$, A. Gehoff ${ }^{3}$, C. Geppert ${ }^{2}$, K. Jung ${ }^{4}$, K. Knobloch $^{1}$, C. Langner ${ }^{5}$,

A. Lugli ${ }^{6}$, I. Nagtegaal ${ }^{7}$, J. Rüschoff ${ }^{3}$, X. Saegert ${ }^{8}$, M. Sarbia ${ }^{9}$, M. Vieth $^{10}$, A. Hartmann ${ }^{2}$

'Pathologisches Institut, Universitätsklinikum Erlangen

${ }^{2}$ Universitätsklinikum Erlangen

${ }^{3}$ Pathologie Nordhessen, Kassel

${ }^{4}$ Universitätsmedizin Göttingen

5Institut für Pathologie, Medizinische Universität Graz, Österreich

${ }^{6}$ Institut für Pathologie, Universität Bern, Schweiz

7Pathologisches Institut, UDC St. Radboud, Nijmegen, Niederlande

${ }^{8}$ Institut de Pathologie, Katholieke Universiteit Leuven, Belgien

${ }^{9}$ Gemeinschaftspraxis für Pathologie und Zytologie München

${ }^{10}$ Institut für Pathologie, Bayreuth

Interobservervariabilität bei der Diagnostik kolorektaler Polypen unter besonderer Berücksichtigung serratierter Läsionen - eine europäische Studie 